\title{
Turbulent flow and heat transfer in eccentric annulus
}

\author{
NIKOLAY NIKITIN ${ }^{1} \uparrow$, HENGLIANG WANG \\ AND SERGEI CHERNYSHENKO ${ }^{2}$
}

\footnotetext{
${ }^{1}$ Institute of Mechanics, Moscow State University, 1 Michurinsky Prospect, 119899 Moscow, Russia

${ }^{2}$ Department of Aeronautics, Imperial College London, Prince Consort Road, London SW7 2AZ, UK
}

(Received 23 September 2008; revised 7 May 2009; accepted 7 May 2009; first published online 18 September 2009)

A detailed statistical analysis of turbulent flow and heat transfer in eccentric annular duct was performed via direct numerical simulations (DNS) with particular emphasis on the needs of turbulence closure models. A large number of flow characteristics such as components of the Reynolds stress tensor, temperature-velocity correlations and some others were obtained for the first time for such kind of a flow. The results of the paper will serve as a benchmark test case for turbulence modelling, specifically for models intended to be used for flows with partly turbulent regimes.

\section{Introduction}

Consider the pressure-gradient-driven fluid flow along the gap between two parallel but eccentric cylinders of different radii, $r_{1}$ and $r_{2}>r_{1}$ (see the sketch in figure $1 a$ ). If eccentricity $e=s /\left(r_{2}-r_{1}\right)$ is large enough, then the width of the gap in the narrow part of the duct $\left(r_{2}-r_{1}\right)-s$ will be considerably less than that in the wide part $\left(r_{2}-r_{1}\right)+s$. Consequently, the fluid velocity in the narrow gap will be less and the local Reynolds number will be significantly less than those in the wide gap. The geometrical parameters may be chosen in such a way that the local Reynolds number in the narrow gap will be too small to sustain turbulence, no matter how large is the global Reynolds number $R e=2\left(r_{2}-r_{1}\right) U_{b} / v$ (here, $2\left(r_{2}-r_{1}\right)$ is the hydraulic diameter and $U_{b}$ is the bulk velocity). In the recent numerical study by Nikitin (2006a), it was indeed shown that two different flow regimes, namely fully turbulent and partly turbulent, may exist at the same Reynolds number but different geometrical configurations (see figure 1).

An eccentric annular duct is a prototype element in a number of engineering applications. Close-packed tubular heat exchangers and coolant channels of nuclear reactor containing clusters of fuel pins are few examples. A considerable experimental and modelling effort has been devoted to determining both the details of the flow field and heat transfer characteristics for such kind of a flow. Deissler \& Taylor (1955) derived an analytical model assuming that the universal velocity profile for circular tube can be applied along lines normal to the bounding walls of the annular duct. Experimental determination of friction factor for relatively large diameter-ratio ducts $\left(\gamma=r_{1} / r_{2}=0.688,0.75,0.875\right)$ was reported by Dodge (1963). Wolffe (1962) measured velocity profiles along lines perpendicular to the outer bounding wall in an annulus with diameter ratio 0.65 and eccentricity 0.46 . A wide-ranging experimental 

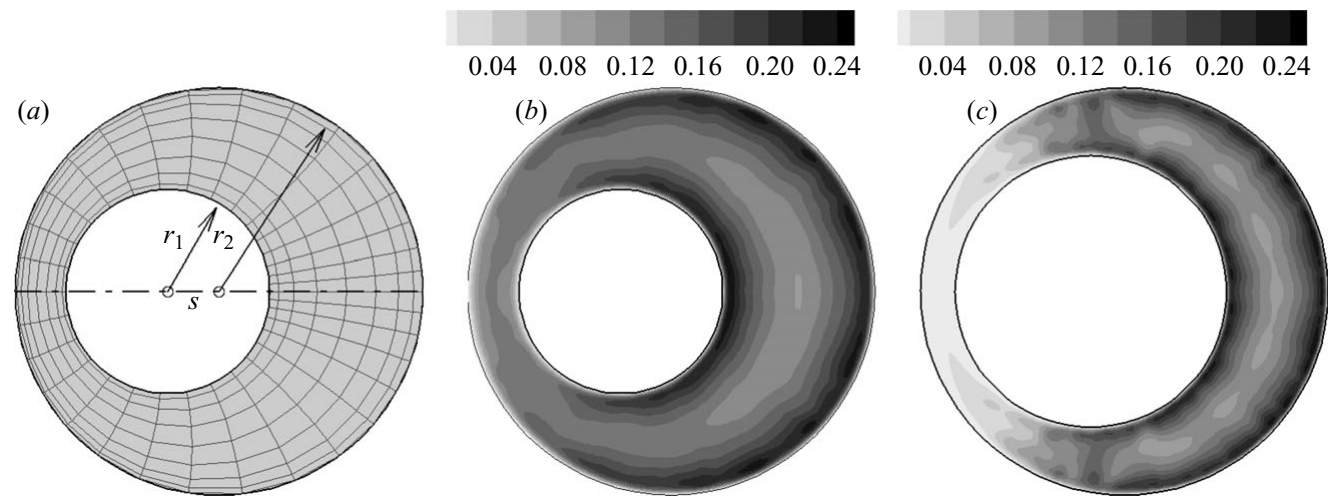

FIGURE 1. (a) Sketch of the duct's cross-section and computational mesh (every 16th grid line is shown in each direction). Contours of r.m.s. velocity fluctuations at $R e=4000$ and $s /\left(r_{2}-r_{1}\right)=1 / 2$ (Nikitin 2006a): (b) fully turbulent flow $\left(r_{1} / r_{2}=1 / 2\right) ;(c)$ partly turbulent flow $\left(r_{1} / r_{2}=2 / 3\right)$.

study aimed at determining friction factor characteristics and details of the flow field was conducted by Jonsson \& Sparrow (1966). The experiments were performed utilizing three annular ducts of different diameter ratios; in each case eccentricity was varied from zero to unity. The experimental study of turbulent flow in a circular pipe containing one or two rods located off-centre was described in Kacker (1973). Mean velocity measurements were obtained and secondary-flow velocities of the order of $1 \%$ of the mean velocity were observed. Some analytical observations using a bipolar coordinate system was presented in Usui \& Tsuruta (1980). Ogino, Sakano \& Mizushina (1987) performed an analysis and measurements of momentum and heat transfer from turbulent flow in an eccentric annulus to inner and outer tube walls. Three components of mean velocity and the corresponding Reynolds shear stresses were measured by Nouri, Umur \& Whitelaw (1993) in concentric and eccentric annulus flows of Newtonian and non-Newtonian fluid.

From a fundamental point of view, turbulent flow in eccentric annular ducts is interesting in several respects. First, it is an ideal model for investigating inhomogeneous turbulent flows, where the conditions of turbulence production vary significantly within the cross-section. Second, it represents a convenient model to study the peculiarities of the flow in the neighbourhood of the laminar/turbulent interface and the related entrainment phenomenon. Third, it may serve as a benchmark test case for turbulence modelling, specifically for models intended to be used for flows with partly turbulent regimes.

The main investigative approach in the present paper is direct numerical simulations (DNS). During the last decades, DNS has been recognized as a powerful and reliable tool for studying the basic physics of turbulent flows. Numerous examples showed that results obtained by DNS are in excellent agreement with experimental findings, if the latter are reliable (see Moin \& Mahesh 1998). In some respects DNS-based studies are even more advantageous than experimental ones since far more detailed information on the flow field structure can be achieved.

However, one major difficulty that arises with a numerical investigation of turbulent flow is the presence of a vast continuous range of relevant scales of motion which must be correctly resolved by numerical simulation. That is why most DNS-based 
works are restricted to relatively low Reynolds number and focus on simple-geometry flows for which accurate and efficient numerical algorithms exist. For wall-bounded turbulent flows, the majority of successful DNS-based studies dealt with the simplest geometry cases such as a plane channel, a flat plate boundary layer, a circular pipe and some other similar cases (see Kim, Moin \& Moser 1987; Spalart 1988; Eggels et al. 1994; Nikitin 1994, 1996).

The turbulent flows listed above are one-dimensional in the mean. Meanwhile, two-dimensional (in the mean) turbulent flows, such as flows in non-circular ducts are interesting not only in engineering but also in fundamental aspects. In particular, they are distinguished by the presence of secondary mean motions in the plane perpendicular to the streamwise flow direction, which are known as secondary motions of Prandtl's second kind.

For an infinitely long straight duct of arbitrary cross-section the incompressible Navier-Stokes equations always have an exact laminar steady solution with only one non-zero streamwise velocity component, which depends on transversal coordinates only. For the eccentric annular pipe considered here, laminar solution is given, for example, by Wilson (1978). Since transversal velocity components are zero in laminar solution, laminar flows in ducts of arbitrary cross-section do not exhibit secondary flows. In turbulent flow the anisotropy of Reynolds stresses in the circumferential direction causes the appearance of a secondary motion in the cross-sectional plane of the duct. Such motions are an intrinsic feature of turbulent flow in non-circular ducts (see Bradshaw 1987). Despite the fact that the secondary velocity usually is only $1 \%-2 \%$ of the streamwise bulk velocity, secondary motions play a significant role, since they result in the cross-stream transfer of momentum, heat and mass (see Demuren \& Rodi 1984; Nikitin \& Yakhot 2005). The development of turbulence closure models that can reliably predict turbulence-driven secondary motions in noncircular ducts is currently not feasible due to the lack of detailed experimental data. Reported DNS-based studies mainly relate to turbulent flows through straight ducts of rectangular cross-section (see Gavrilakis 1992; Huser \& Biringen 1993; Nikitin 1997). The recent papers by Nikitin \& Yakhot (2005) and Voronova \& Nikitin (2007), where detailed DNS results of turbulent flow in elliptical ducts were reported, are the rare exception.

Regarding the eccentric annulus flow, first DNS studies were performed by Nikitin (2006a) and Ninokata et al. (2006). Nikitin (2006a) considered two geometrical cases with diameter ratios $\gamma=1 / 2$ and $2 / 3$ at Reynolds number $R e=4000$ and eccentricity $e=1 / 2$ in both cases. Two distinct flow regimes, fully turbulent and partly turbulent, were detected in these cases. Mean velocity, turbulence intensity and secondary-motion patterns were obtained in simulations. Ninokata et al. (2006) reported preliminary DNS results for the flow at $R e=12100$ in the duct with $\gamma=e=1 / 2$.

The aim of the present work is to provide a comprehensive DNS-based data of turbulent flow and heat transfer in eccentric annulus when the turbulent flow occupies the entire duct's cross-section.

\section{Formulation and numerical method}

We consider non-isothermal flow of an incompressible viscous fluid through an eccentric annular duct ( $x, y$ and $z$ are Cartesian coordinates)

$$
G=\left\{(x, y, z):\left(x-x_{1}\right)^{2}+y^{2} \geqslant r_{1}^{2} \cap\left(x-x_{2}\right)^{2}+y^{2} \leqslant r_{2}^{2}, \quad|z|<\infty\right\} .
$$


The flow is governed by the Navier-Stokes and energy equations (with thermal dissipation neglected)

$$
\begin{gathered}
\frac{\partial \boldsymbol{u}}{\partial t}=\boldsymbol{u} \times \operatorname{rot} \boldsymbol{u}-v \operatorname{rot} \operatorname{rot} \boldsymbol{u}-\operatorname{grad}\left(\frac{p}{\rho}+\frac{|\boldsymbol{u}|^{2}}{2}\right)-\boldsymbol{k} \frac{1}{\rho} \frac{\mathrm{d} \hat{p}}{\mathrm{~d} z}, \\
\rho c_{p}\left(\frac{\partial T}{\partial t}+\operatorname{div}(T \boldsymbol{u})\right)=\kappa \operatorname{div} \operatorname{grad} T,
\end{gathered}
$$

subjected to the incompressibility constraint

$$
\operatorname{div} \boldsymbol{u}=0 .
$$

Here, $\boldsymbol{u}$ is the velocity field, $p$ is the pressure, $\rho$ is the fluid density, $v$ is the kinematic viscosity, $\kappa$ and $c_{p}$ are the thermal conductivity and specific heat of the fluid, $\boldsymbol{k}$ is the unit vector in the $z$-direction. We imply the no-slip boundary condition at the rigid duct's walls and periodic boundary conditions in the streamwise $z$-direction. The inner and outer cylinders are assumed to be isothermal with the temperatures $T_{1}$ and $T_{2}$, respectively. In (2.3), $T$ stands for the temperature difference between the fluid particle and the inner cylinder wall. Thus, $T=0$ on the inner wall and $T=T_{2}-T_{1}$ on the outer one. In (2.2), we split the pressure gradient into two terms, where, due to the implied periodicity, the first $(\operatorname{grad} p)$ does not contribute to the overall pressure drop. In order to maintain a constant flow rate $Q_{0}$, the mean pressure gradient $\mathrm{d} \hat{p} / \mathrm{d} z$ is determined at each time instant from the constraint

$$
\iint_{\Omega} u_{z}(x, y, z, t) \mathrm{d} x \mathrm{~d} y=Q_{0}=\text { constant. }
$$

In (2.5), $\Omega$ denotes the duct's cross-section, and the integral does not depend on $z$ due to incompressibility.

The problem is solved using curvilinear bipolar coordinates $(\xi, \eta)$ introduced in the cross-sectional plane of the duct

$$
x=-\lambda(\xi, \eta) \sinh \xi, \quad y=\lambda(\xi, \eta) \sin \eta, \quad \lambda(\xi, \eta)=\frac{c}{\cosh \xi-\cos \eta},
$$

where $c=\left[\left(r_{2}^{2}-r_{1}^{2}-s^{2}\right)^{2} /\left(4 s^{2}\right)-r_{1}^{2}\right]^{1 / 2}$. The bipolar coordinates vary in the range $0 \leqslant \eta \leqslant 2 \pi$ and $\xi_{1} \leqslant \xi \leqslant \xi_{2}$, where $\xi_{1}=\ln \left[\left(1+c^{2} / r_{1}^{2}\right)^{1 / 2}-c / r_{1}\right]$ and $\xi_{2}=$ $\ln \left[\left(1+c^{2} / r_{2}^{2}\right)^{1 / 2}-c / r_{2}\right]$. The surfaces $\xi=\xi_{1}$ and $\xi=\xi_{2}$ coincide with the surfaces of the inner and outer cylinders, respectively. The plane $\eta=0$ intersects the duct along the wide part of the gap, and the plane $\eta=\pi$, along its narrow part.

Numerical solution to the system (2.2)-(2.4) is obtained by using the method described in Nikitin (2006b). It exploits a second-order-accurate conservative finitedifference scheme of spatial discretization and a third-order-accurate semi-implicit Runge-Kutta method for time advancement. The Poisson equation for the pressure is solved iteratively by using the fast Fourier transform in the $z$-direction and a combination of the conjugate-gradient method and the fast cyclic reduction method of Swarztrauber (1974) in the $(\xi, \eta)$-plane.

The numerical procedure can be described as follows. Starting with some initial three-dimensional velocity field, the governing equations are integrated in time until a statistically steady state is reached. Then, the mean flow and turbulence statistical quantities are obtained by further time-advancing and averaging both in time and along the homogeneous $z$-direction. The flow in a limiting turbulent regime is statistically symmetric about the plane $y=0$. Statistical sampling is further increased 


$\begin{array}{cc}\text { Wide gap } & \text { Narrow gap } \\ 1.2 & 0.39 \\ 1.1 & 0.19 \\ 1.4 & 3.1 \\ 3.6 & 4.2 \\ 15.1 & 10.5 \\ 14.2 & 9.8\end{array}$

TABLE 1. Grid resolutions.

by the averaging over the two symmetric parts of the flow. The result of this averaging procedure is that the mean fields depend on $\xi$ and $\eta$ only and are symmetric about the plane $y=0$. In what follows, the averaging is denoted by angular brackets; a quantity $f^{\prime}$ means an instantaneous fluctuation of $f$, i.e. $f=\langle f\rangle+f^{\prime}$.

In this paper, we present the results obtained at Reynolds number $R e=8000$ and Prandtl number $\operatorname{Pr}=\alpha / \nu=1\left(\alpha=\kappa /\left(\rho c_{p}\right)\right.$ is the thermal diffusivity of the fluid) for the duct with the diameter ratio $\gamma=0.5$ and the eccentricity $e=0.5$. Simulation was performed with a streamwise period $L_{z}=12.56 \delta\left(\delta=r_{2}-r_{1}\right.$ is the mean clearance $)$ on a mesh with $128 \times 512 \times 256$ grid nodes in the $\xi$-, $\eta$ - and $z$-directions, respectively. The non-uniform grid in the both cross-sectional coordinates $\xi, \eta$ was constructed ensuring node clustering in the most dynamically significant regions of the flow, in the neighbourhood of rigid walls and in the wide gap of the duct (see figure $1 a$ ). The grid sizes in the widest $(\eta=0)$ and the narrowest $(\eta=\pi)$ regions of the flow domain in the vicinity of the inner and outer cylinders are given in the table 1 . Local viscous length scales are used in each case. The data in table 1 demonstrate adequacy of the grid to represent the smallest scales in the wall-bounded turbulent flow at given Reynolds number.

In what follows, all data are presented in the non-dimensional form with the bulk velocity $U_{b}$, the mean clearance $\delta$ and the wall-temperature difference $T_{2}-T_{1}$ as the velocity, length and temperature scales, respectively. Flow statistics presented in the paper were obtained by the time averaging over the interval $T_{a v}=2817$. The adequacy of the time-averaging interval was validated by comparing with the statistics obtained with different intervals: $T_{a v}=1428,1045,645$. For the most characteristics shown in the paper, the chosen interval is sufficiently long. Few cases, where the time-averaging interval is not fully adequate are discussed below.

In order to provide the broadest possible perspective, the details of the flow and temperature fields over the cross-sectional plane are displayed in several different ways. The first is in the form of contour maps, while the second and third show profiles along the lines normal to the bounding walls, normalized either by conventional scales, or by the local wall variables. The set of nine normal-to-walls lines $\eta=\eta_{j}, j=0, \ldots, 8$ chosen for the data presentation are equally spaced in the circumferential direction within the upper half of the cross-section. The averaged flow is symmetric about the plane $y=0$, therefore the lower-half distributions are not shown. The location of the nine lines within the duct's cross-section is shown in figure 2 and the values of $\eta_{j}$ are given in table 2. For simplicity we call the profiles along the lines $\eta=\eta_{j}$ as radial profiles, although the lines $\eta=$ constant are not straight lines except the $\eta=0$ and $\eta=\pi$. The radial profiles at each $\eta$ are given as functions of distance $d$ measured 


$\begin{array}{cccccccccc}j & 0 & 1 & 2 & 3 & 4 & 5 & 6 & 7 & 8 \\ \eta_{j} / \pi & 0 & 0.0723 & 0.1489 & 0.2348 & 0.3329 & 0.4510 & 0.6053 & 0.7903 & 1.0\end{array}$

TABLE 2. Values of $\eta$ along the data presentation lines.

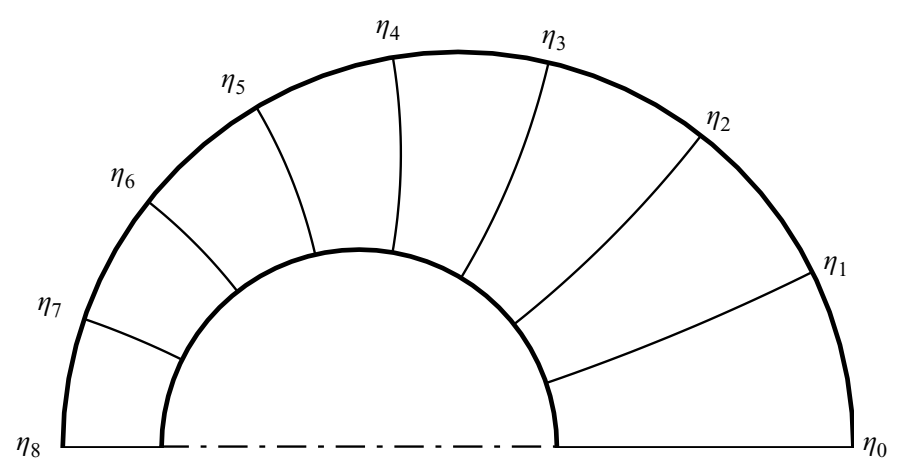

FigURE 2. Location of lines chosen for data presentation.

along the line from the midpoint between the cylinder walls,

$$
d=\int_{\xi_{1}}^{\xi} \lambda(\xi, \eta) \mathrm{d} \xi-\frac{h}{2}, \quad h(\eta)=\int_{\xi_{1}}^{\xi_{2}} \lambda(\xi, \eta) \mathrm{d} \xi
$$

Here, $h(\eta)$ is the local duct's clearance. When the data are normalized by the local wall variables $u_{\tau}=\sqrt{\tau_{w} / \rho}$ and $l_{\tau}=\nu / u_{\tau}$ defined by the local wall shear stress $\tau_{w}$ either on the inner or the outer wall, the distance to the wall $d^{+}$is measured along straight lines perpendicular to the respective wall. Normalization by the wall variables is denoted by a superscript + .

Circumferential variation of flow characteristics is presented in the paper in the form of functions of bipolar coordinate $\eta$. Each value of $\eta$ corresponds to different angular coordinates $\theta_{1}$ and $\theta_{2}$ on the inner and outer cylinder walls. The correspondence between $\theta_{1}, \theta_{2}$ and $\eta$ is given in figure 3 .

\section{Flow field simulation results}

\subsection{Wall friction and mean velocity}

The law of friction in eccentric ducts is an issue of controversial discussions. The most thorough measurement of the friction factor $C_{f}=\bar{\tau}_{w} / 0.5 \rho U_{b}^{2}$ was performed by Jonsson \& Sparrow (1966). In the definition of friction factor, $\bar{\tau}_{w}$ is the wall friction averaged along both bounding walls. It is related to the mean pressure gradient by

$$
\bar{\tau}_{w}=\frac{\delta}{2}\left(-\frac{\mathrm{d} \hat{p}}{\mathrm{~d} z}\right) .
$$

Jonsson \& Sparrow (1966) analysed the flows in eccentric ducts with diameter ratios $\gamma=0.281,0.561$ and 0.75 . For each of these ducts, eccentricity varied from zero to unity. The Reynolds number range of the investigation extended from 18000 to 180000. The friction-factor information was correlated by a power-law relationship of the type $C_{f}=C / R e^{n}$. It was found that an $n$ value of 0.18 provided the most 


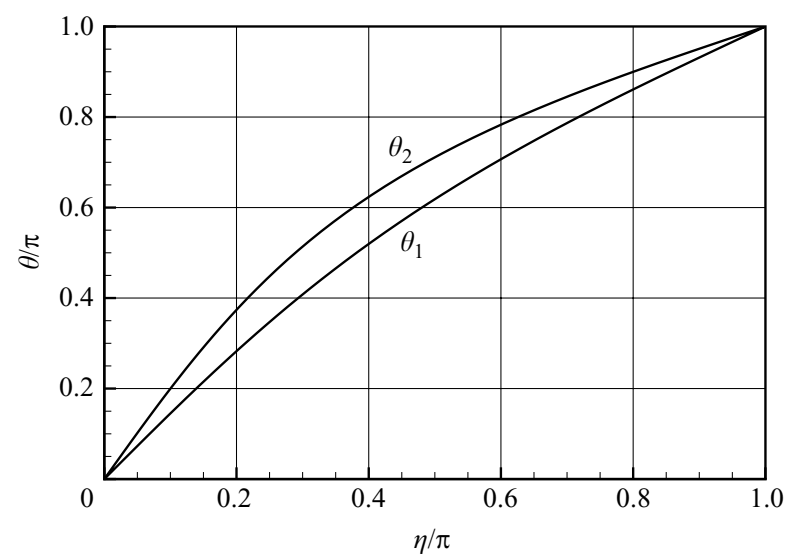

FIGURE 3. Angular coordinates on the inner $\theta_{1}$ and the outer $\theta_{2}$ cylinder walls as functions of bipolar coordinate $\eta$.

satisfactory correlation for all Reynolds numbers, diameter ratios and eccentricities. Different Reynolds number exponents, larger than 0.18 were suggested in Deissler \& Taylor (1955) and in Dodge (1963). Regarding the value of $C$, it depends both on $\gamma$ and $e$. In general, for a fixed Reynolds number and diameter ratio, the friction factor decreases with increasing eccentricity. Kacker (1973) experimentally deduced the relation $C_{f}=0.0445 R e^{-0.196}$ for the eccentric annulus with $\gamma=0.176$ and $e=0.475$ as well as for the pipe with two rods located off-centre. Nouri et al. (1993) measured the friction factor in a duct with $\gamma=0.5$ for three values of eccentricities, $e=0,0.5$ and 1. They found that at $R e=26,600$ the friction factor in the concentric annulus was about $8 \%$ larger than that for smooth circular pipe. In the eccentric ducts $C_{f}$ was lower than in the concentric one by $8 \%$ and $22.5 \%$ for $e=0.5$ and $e=1$, respectively.

In our simulation, the friction factor was $C_{f}=8.28 \times 10^{-3}$. This value is almost four times larger than that in the laminar flow at the same Reynolds number, where $C_{f}=2.21 \times 10^{-3}$ and is very close to the friction factor for a circular pipe as predicted by the Blasius formula $C_{f}=0.0791 R e^{-0.25}$; the difference is $-1.0 \%$. Thus, our data agree with the Nouri et al. (1993) prediction for the eccentric duct.

Distribution of the local wall shear stress $\tau_{w, z}$ along the inner and the outer walls is shown in figure 4. Shear stress on the inner wall is $10 \%-15 \%$ higher than that on the outer wall everywhere along the perimeter. Laminar flow distributions are also shown in figure 4 giving an immediate idea of rising the friction force in turbulent flow.

In what follows, we use the upper-case letters to denote mean values of the pressure and velocity components: $P \equiv\langle p\rangle, U_{z} \equiv\left\langle u_{z}\right\rangle$ and so on. Mean velocity distribution over the duct's cross-section is shown in figures 5 and 6 and compared with that in the laminar flow. The latter is a solution to the equation

$$
\frac{v}{\lambda^{2}}\left(\frac{\partial^{2} U_{z}}{\partial \xi^{2}}+\frac{\partial^{2} U_{z}}{\partial \eta^{2}}\right)-\frac{1}{\rho} \frac{\mathrm{d} \hat{p}}{\mathrm{~d} z}=0 .
$$

Radial profiles in the laminar flow have almost parabolic shape for each $\eta$, while in the turbulent flow the profiles have typical turbulent shape. The mean velocity distribution in the turbulent flow is significantly flattened out both in the radial and in the circumferential direction compared with that in the laminar flow. Maximal 


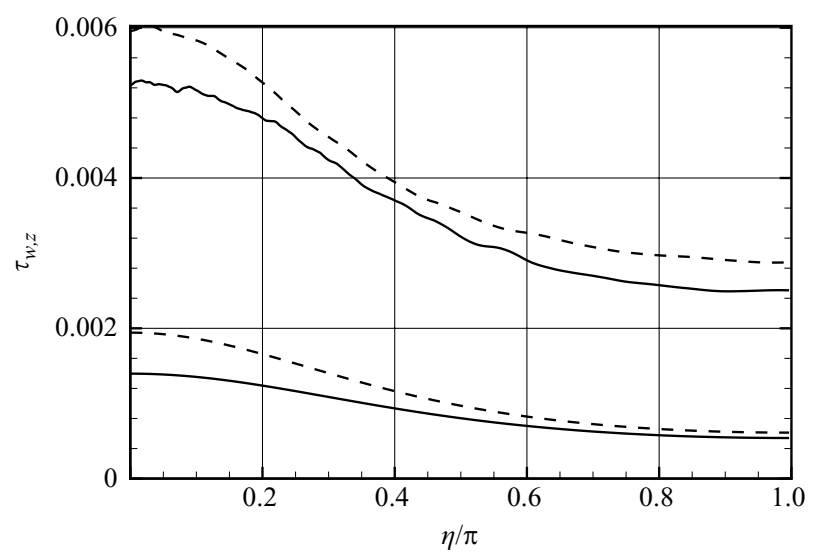

FIGURE 4. Local wall shear stress distribution in laminar flow (lower lines) and turbulent flow (upper lines). Dashed lines: inner wall; solid lines: outer wall.
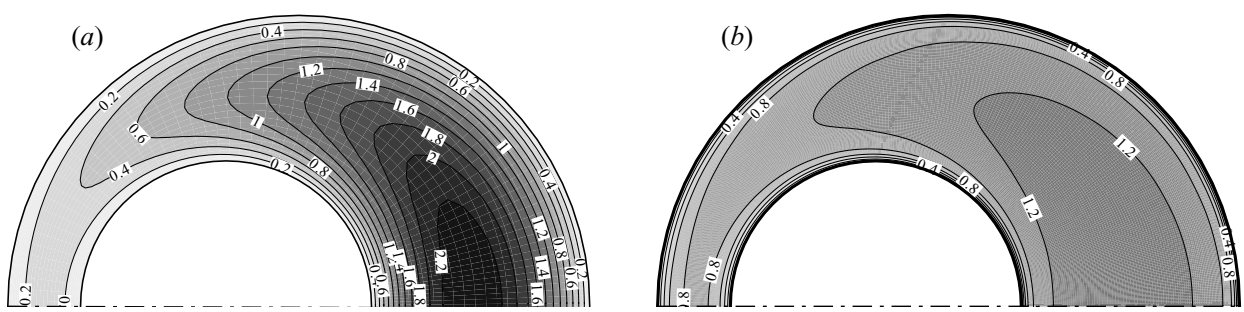

FIGURE 5. Mean velocity contours. (a) Laminar flow; $(b)$ turbulent flow.
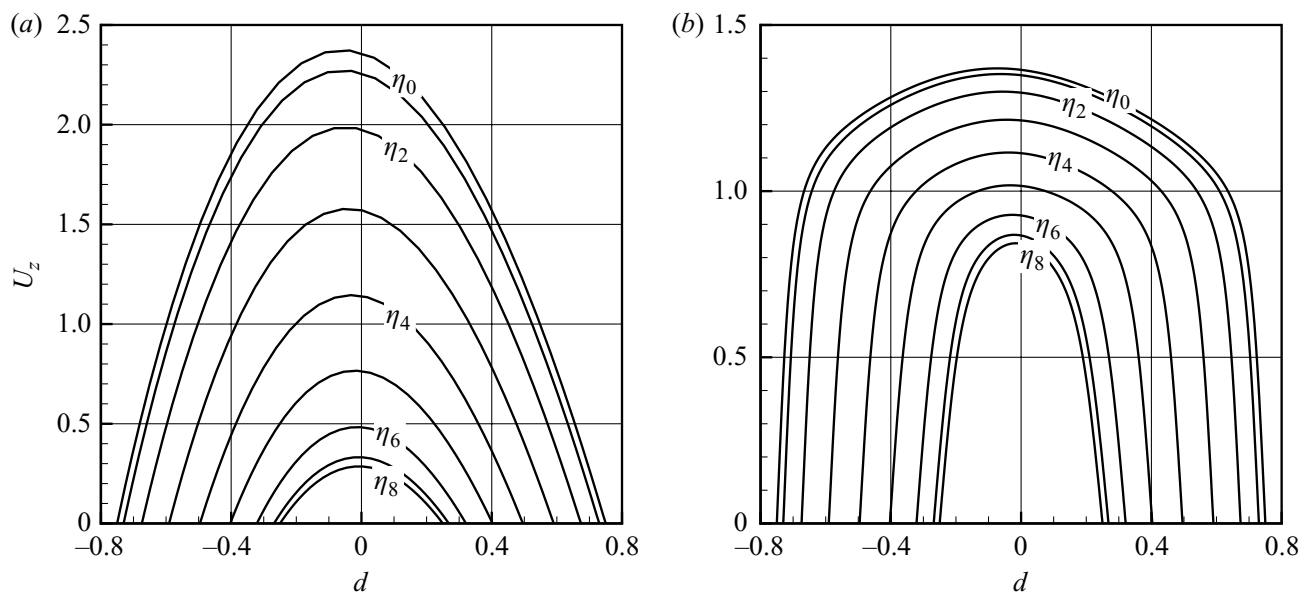

FiguRE 6. Mean velocity profiles. (a) Laminar flow; $(b)$ turbulent flow.

velocities in the wide and in the narrow gaps are 1.36 and 0.85 , respectively, in the turbulent flow and 2.37 and 0.29 in the laminar flow. The profiles are slightly inclined towards the inner wall in the both flows: the maximum velocity occurred at locations closer to the inner wall. The same inclination of turbulent velocity profile in the wide gap was observed experimentally by Nouri et al. (1993) in the duct with the 

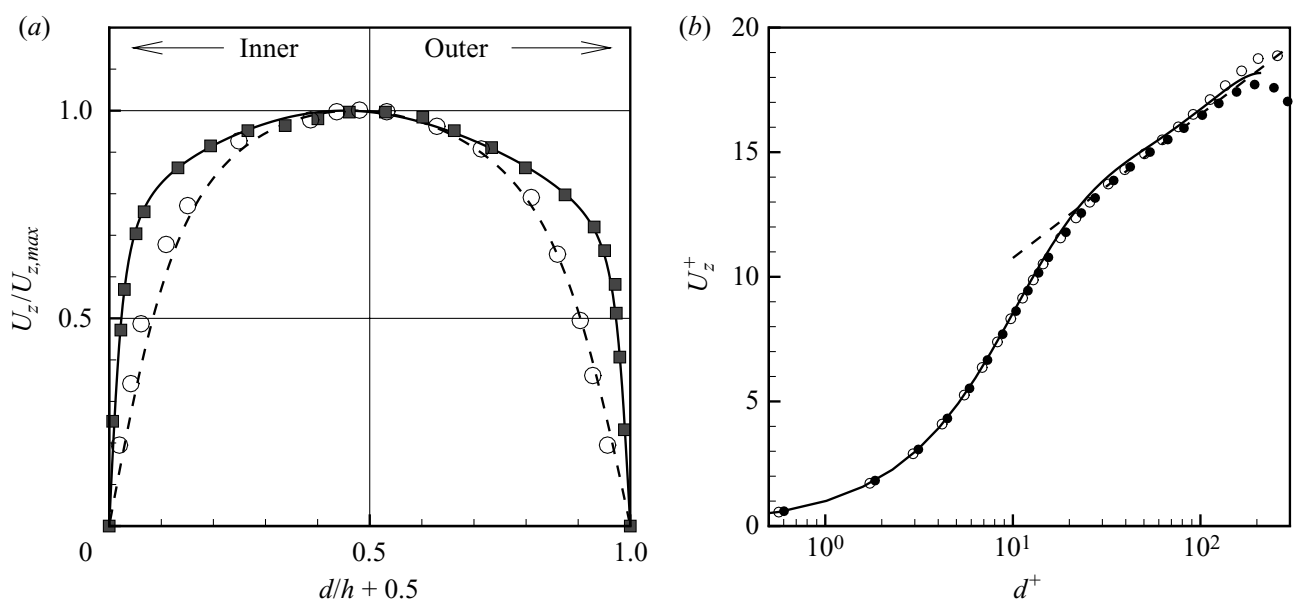

FIGURE 7. Mean-velocity profiles: ( $a$ ) comparison of present simulation (lines) with experiment Nouri et al. (1993) (symbols), solid line and closed symbols correspond to the wide gap $(\eta=0)$, dashed line and open symbols correspond to the narrow gap $(\eta=\pi) ;(b)$ wide-gap profiles: closed symbols correspond to inner profile, open symbols correspond to outer profile, solid line: mean velocity in a plane channel flow at $R e_{\tau}=211$, dashed line: $U_{z}^{+}=2.5 \log d^{+}+5.0$.

same eccentricity and diameter ratio and close Reynolds number $R e=8900$. Mean velocity profiles of present simulation in the wide and narrow gaps are compared with experimental data of Nouri et al. (1993) in the figure 7(a). The agreement is very good in both cases.

It is a common practice to represent turbulent velocity profiles in duct flow in terms of the so-called law of the wall. The validity of the universal logarithmic mean velocity distribution

$$
U_{z}^{+}=A \log d^{+}+B
$$

in eccentric annulus is discussed in a number of investigations. In their analysis Deissler \& Taylor (1955) assumed that the law of the wall with the constants $A=2.78$ and $B=3.8$ is valid everywhere in the duct along the lines perpendicular to the walls of the annulus. The measurements of Jonsson \& Sparrow (1966) did not substantiate this assumption. They found that in a concentric annulus the inner profiles lie substantially lower than the outer ones. After inspecting three different diameter ratios they suggested expression (3.3) with constants $A=2.44, B=4.9$ for inner profiles and $A=2.56, B=4.9$ for outer profiles. In eccentric case a fair agreement with the universal logarithmic distribution was observed only for outer profiles when $\theta_{2}$ is no larger than $\pi / 2$ or $2 \pi / 3$. At larger angles experimental points lie significantly higher than the universal line. For the inner profiles the universal logarithmic distribution in the wide gap was observed only for the largest diameter ratio $\gamma=0.75$ duct. A similar conclusion that the law of the wall in eccentric annulus is valid for the outer wall except in the smallest gap, but the near-inner-wall flow is not represented by a logarithmic region particularly in the smallest gap, was derived by Nouri et al. (1993).

In our simulation, the inner and outer profiles in the widest gap are very close to each other down to about $d^{+}=100^{+}$, when normalized by the corresponding local-wall scales (see figure $7 b$ ). In the region $30<d^{+}<100$ they both are described reasonably well by the correlation $U_{z}^{+}=2.5 \log d^{+}+5.0$. Also shown in figure $7(b)$ is 

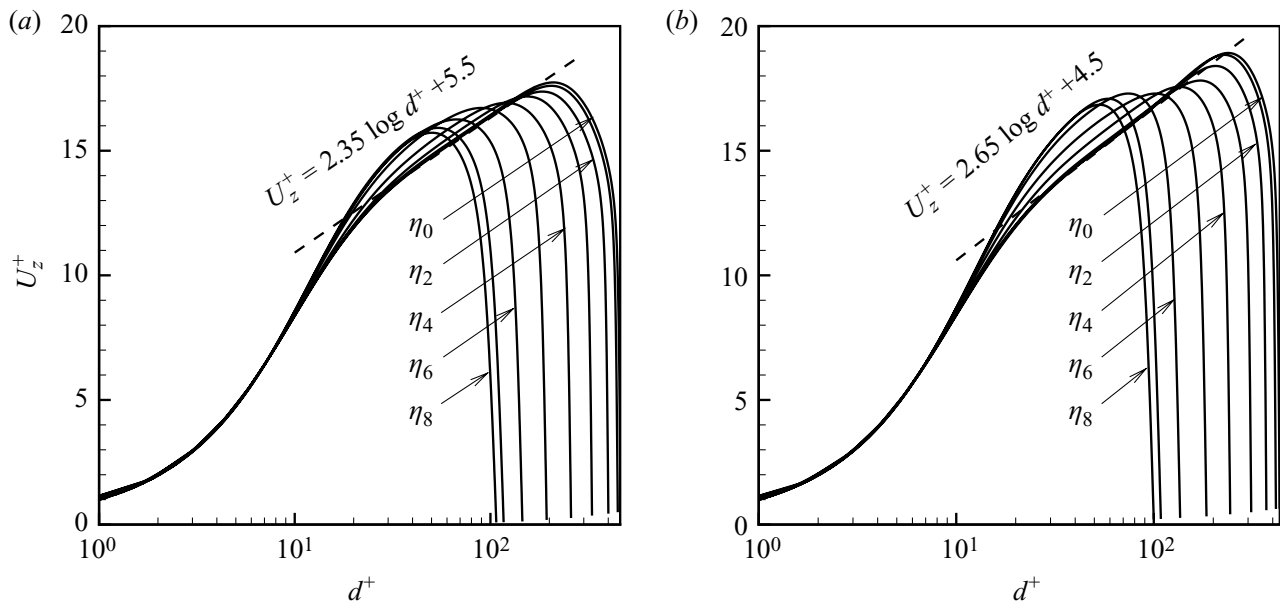

FIGURE 8. Mean velocity profiles. (a) Inner profiles; $(b)$ outer profiles.

mean velocity profile obtained by Gilbert \& Kleiser (1991) in DNS of plane channel flow at $R e_{\tau}=211$. It is taken from the DNS database of turbulence and heat transfer available online (http://www.thtlab.t.u-tokyo.ac.jp/DNS/dns_database.html).

Mean velocity profiles $U_{z}^{+}\left(d^{+}\right)$along the lines $\eta=\eta_{j}$ are shown in figure 8 . To exclude possible effect of the small-scale irregularities in the $\tau_{w}$ distribution, the wall shear stress for each radial profile $\eta_{j}$ was averaged over the interval $\left[0.5\left(\eta_{j-1}+\right.\right.$ $\left.\left.\eta_{j}\right), \quad 0.5\left(\eta_{j}+\eta_{j+1}\right)\right]$. Although the Reynolds number in present simulations was significantly lower than that in the cited experimental investigations, our results are in good qualitative agreement with experiment. The logarithmic regions in the velocity profiles may be detected in the wide gap, $\eta \leqslant \eta_{3}$, while the narrow-gap profiles lie substantially higher than the logarithmic line. The logarithmic distribution with constants $A=2.65$ and $B=4.5$ is clearly visible in the outer profiles. The logarithmic region in the inner profiles is less pronounced and has less steep slope. The corresponding constants are $A=2.35$ and $B=5.5$.

Systematic deviation of the narrow-gap profiles from the logarithmic line in our case may be attributed to a small local Reynolds number effect. Distribution of the local Reynolds number $R e_{\text {local }}$ along circumferential direction is shown in figure 9. $R e_{\text {local }}(\eta)$ is based on the average velocity along a line $\eta=$ constant and on the local clearance of the duct $h(\eta)$ defined in (2.7). Dashed line in the figure 9 presents distribution of the Reynolds number $\operatorname{Re}_{\tau, l o c a l}(\eta)$ based on the half of the local duct's clearance and the local friction velocity (calculated by the wall shear stress, averaged over two walls). Reynolds number $R e_{\text {local }}$ decreases from 7050 in the wide gap down to 1300 in the narrow gap. In this section $R e_{\tau, \text { local }}$ is as small as 52 which is below the smallest $R e_{\tau}=64$ of turbulence existence in a plane channel flow found by Tsukahara et al. (2005).

\subsection{Reynolds stresses}

Distribution of Reynolds stresses $\left\langle u_{i}^{\prime} u_{j}^{\prime}\right\rangle$ over the duct cross-section is shown in figure 10 in the form of radial profiles. The most of the data shown in figure 10 are averaging independent. This, however, is not fully true for the statistics reflecting the azimuthal inhomogeneity of the flow, i.e. $\left\langle u_{\xi}^{\prime} u_{\eta}^{\prime}\right\rangle$ and $\left\langle u_{\eta}^{\prime} u_{z}^{\prime}\right\rangle$ (in azimuthally homogeneous flow, such as the flow in concentric annulus, these statistics are zero). The corresponding profiles should be treated with a certain care. The idea, how 


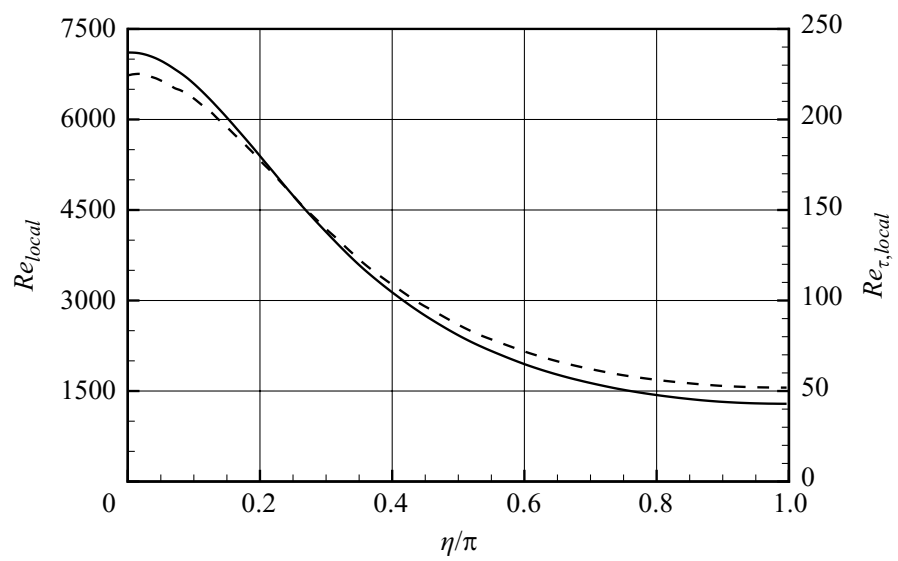

FIGURE 9. Local Reynolds number distribution. Solid line: $R e_{l o c a l}$; dashed line: $R e_{\tau, l o c a l}$.

different statistics depend on the time-averaging interval $T_{a v}$ may be obtained from figure 11. In this figure, the profiles of $\left\langle u_{z}^{\prime} u_{z}^{\prime}\right\rangle$ and $\left\langle u_{\xi}^{\prime} u_{\eta}^{\prime}\right\rangle$ (the latter characteristic is the most sensitive to time-averaging interval) are shown for one particular radial section, $\eta=\eta_{4}$, but for different time-averaging intervals: $T_{a v}=645,1045,1428$ and 2817. One can see almost complete coincidence of all profiles of $\left\langle u_{z}^{\prime} u_{z}^{\prime}\right\rangle$ proving the adequacy of averaging, while some quantitative (but not qualitative) modifications are present in the $\left\langle u_{\xi}^{\prime} u_{\eta}^{\prime}\right\rangle$ profiles for different $T_{a v}$.

The shape of the normal stresses $\left\langle u_{i}^{\prime} u_{i}^{\prime}\right\rangle$ is fairly predictable in the most part of the duct cross-section. Normal stresses exhibit a typical behaviour peculiar to wall-bounded turbulent flows with maximal values in the near-wall region. The only exception is the atypical increase in the intensity of circumferential velocity fluctuations in the narrow part of the duct. Shear stress $\left\langle u_{\xi}^{\prime} u_{z}^{\prime}\right\rangle$ is also typical to wall-bounded flows. Its shape may be explained by considering wall-normal velocity fluctuations acting across the longitudinal mean velocity gradient: a positive wallnormal velocity fluctuation leads to a negative fluctuation of the $u_{z}$ near the inner cylinder wall, where $\partial U_{z} / \partial \xi$ is positive, and to a positive fluctuation of the $u_{z}$ near the outer cylinder wall, where $\partial U_{z} / \partial \xi$ is negative. As a result, shear stress $\left\langle u_{\xi}^{\prime} u_{z}^{\prime}\right\rangle$ is negative in the inner region and positive in the outer one. The analogous consideration is valid for the explanation of the shape of the $\left\langle u_{\eta}^{\prime} u_{z}^{\prime}\right\rangle$. Circumferential gradient of the mean velocity, $\partial U_{z} / \partial \eta$ is negative everywhere in the upper half of the duct crosssection, thus, a positive velocity fluctuation of the $\eta$-component leads to a positive fluctuation of the $z$-component and vice versa. Hence, $\left\langle u_{\eta}^{\prime} u_{z}^{\prime}\right\rangle$ is positive everywhere in the upper half of the duct and negative in the lower half.

\subsection{Quadrant analysis and streaky structures}

The qualitative similarity in the shape of the wide- and narrow-gap velocity statistics shown in the figures 8 and 10 suggests that essentially the same physics lies behind the turbulence in each radial section of the duct. This supposition gets further support by quadrant analysis of the Reynolds shear stresses in different parts of the duct. The quadrant analysis, proposed in Willmarth \& Lu (1972) and Brodkey, Wallace \& Eckelmann (1974), provides the detailed information on the contribution of different flow events to the turbulence production. The analysis divides the Reynolds shear stress into four categories $Q_{1}-Q_{4}$ according to the signs of longitudinal $u_{z}^{\prime}$ and wallnormal $u_{n}^{\prime}$ velocity fluctuations. The contribution to the Reynolds shear stress from 

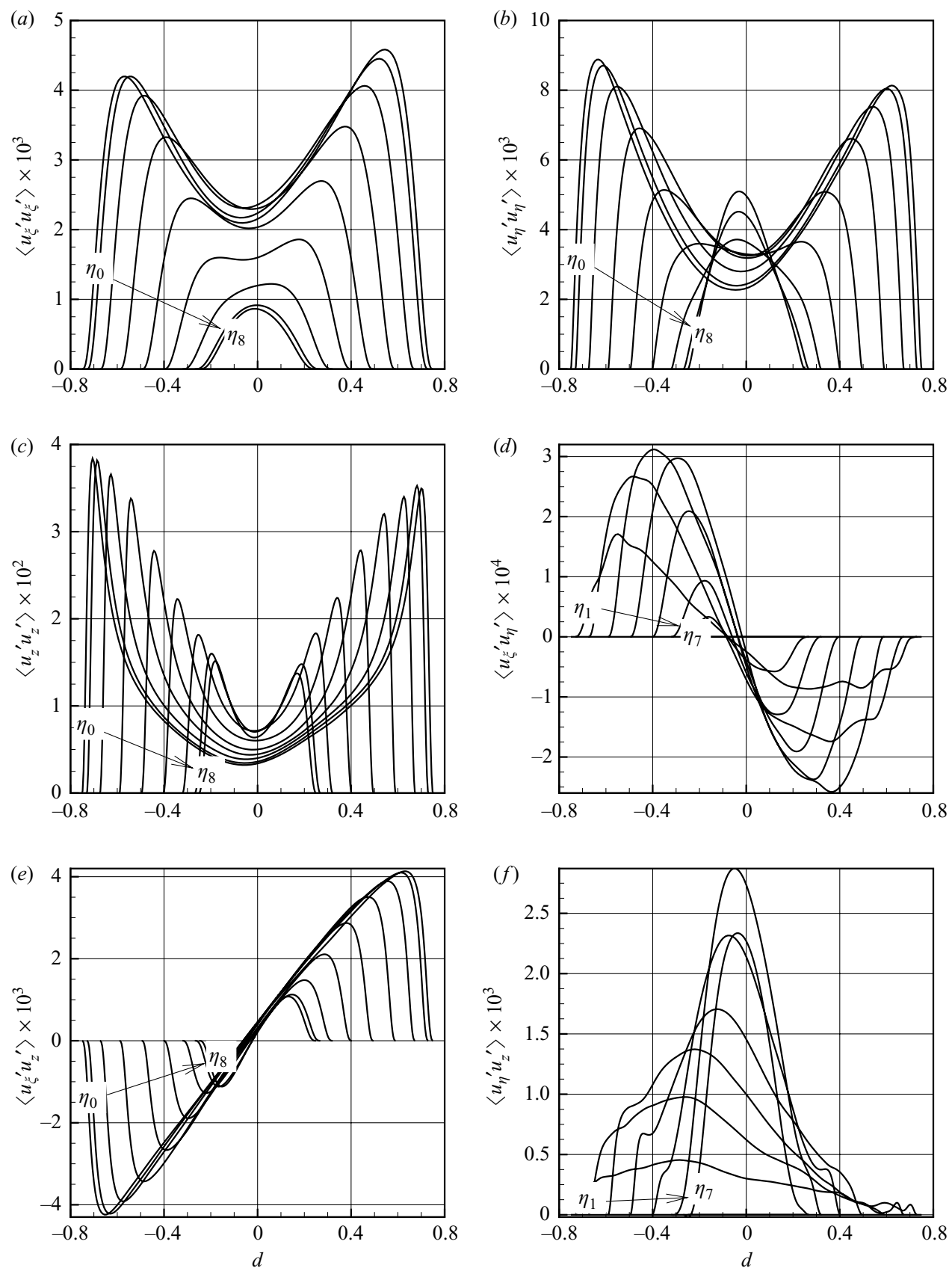

FiguRE 10. Profiles of Reynolds stresses components.

each quadrant in the $\left(u_{z}^{\prime}, u_{n}^{\prime}\right)$ plane is shown in figure 12. The data for the widest and the narrowest gap are given in figure 12 and compared with corresponding distributions in plane channel flow. The quadrant analysis in the present study was conducted by analysing a single instantaneous flow field, thus, no time averaging and only averaging over the $z$-direction was applied. Nevertheless, the data presented in figure 12 demonstrate very similar, and not only qualitative, but also quantitative 

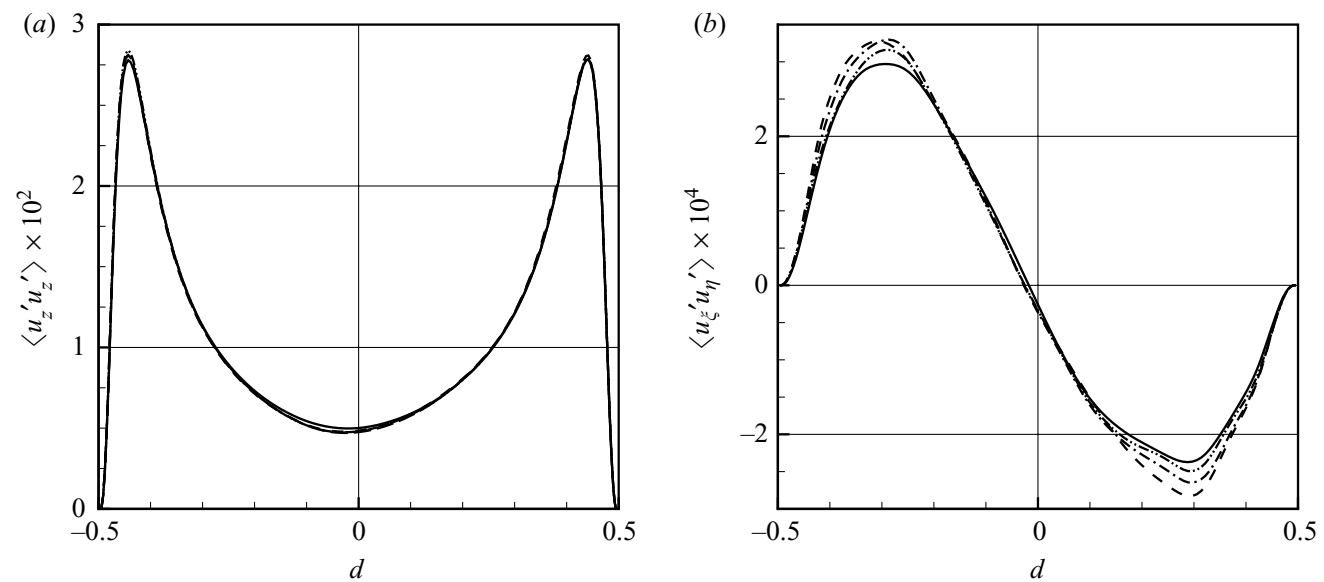

FiguRE 11. Radial profiles of Reynolds stresses tensor components $(a)\left\langle u_{z}^{\prime} u_{z}^{\prime}\right\rangle$ and $(b)\left\langle u_{\xi}^{\prime} u_{\eta}^{\prime}\right\rangle$ at section $\eta=\eta_{4}$ obtained with different time-averaging intervals. Dashed, dash-dotted, dash-dot-dotted and solid lines correspond to $T_{a v}=645,1045,1428$ and 2817, respectively.
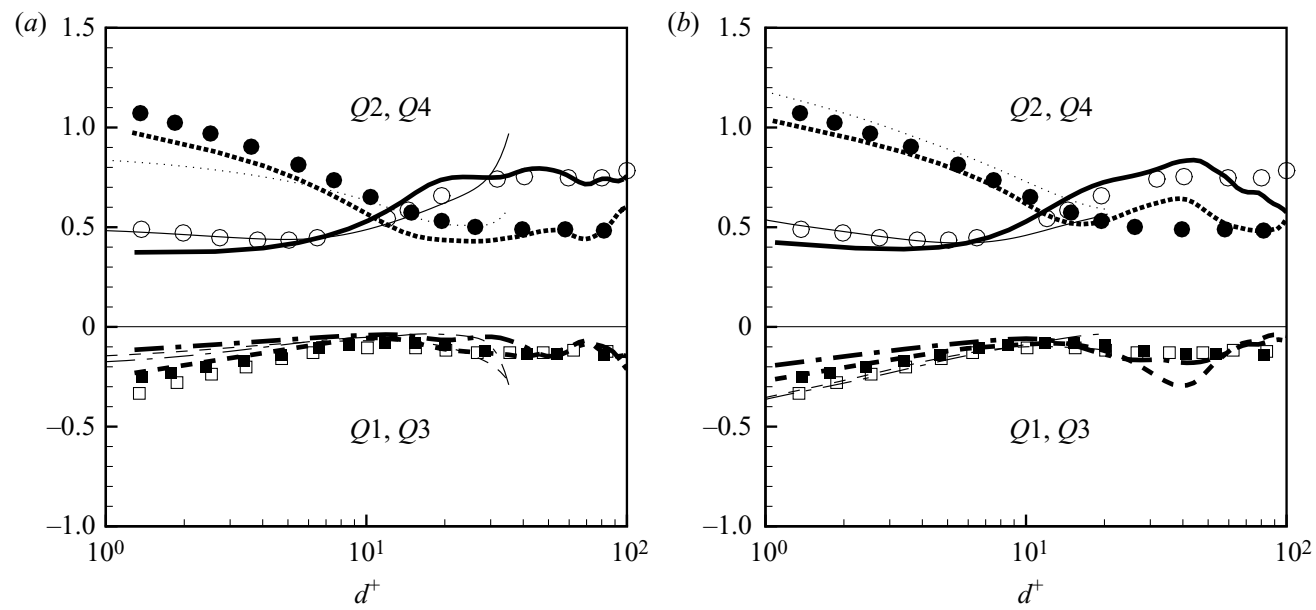

FiguRE 12. Near-wall distribution of the Reynolds shear stress $\left\langle u_{\xi}^{\prime} u_{z}^{\prime}\right\rangle$ from each quadrant normalized by the local mean shear stress: $(a)$ inner cylinder; $(b)$ outer cylinder. Thick lines: wide gap; thin lines: narrow gap; dashed: first quadrant; solid: second quadrant; dash-dotted: third quadrant; dotted: fourth quadrant. Symbols, plane channel flow at $R e_{\tau}=180$ (Kim et al. 1987): $\square$, first; O, second; $\mathbf{n}$, third; $\bullet$, fourth quadrant.

behaviour in both wide and narrow gaps of the eccentric annulus and in plane channel flow. This behaviour is characterized by the dominance of so-called sweep events $\left(Q_{4}\right)$ in the immediate vicinity of the wall $\left(d^{+} \lesssim 12\right)$ which is replaced by the ejection events $\left(Q_{2}\right)$ at further distance from the wall.

The self-sustaining process of turbulence regeneration in wall-bounded flows is associated with the existence of longitudinal streaks in near-wall velocity field. The streaks are most pronounced in the region of buffer layer of the mean velocity profile. The footprints of streaks can be detected on the wall surface in the field of velocity gradient. Figure 13 presents instantaneous distribution of the fluctuation part of the longitudinal velocity gradient $\partial u_{z}^{\prime} / \partial n$ on the surfaces of the inner and outer cylinders. 

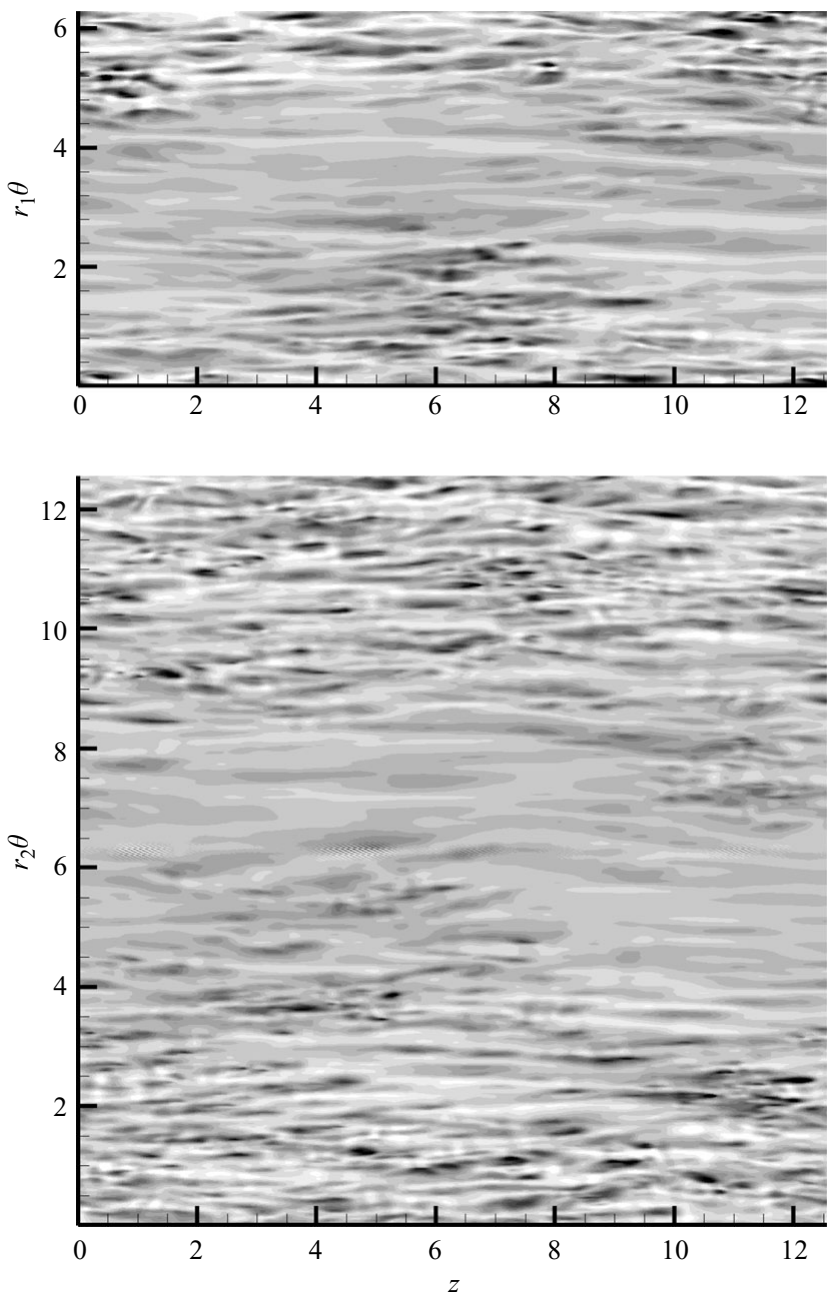

FIGURE 13. Footprints of longitudinal velocity streaks on the cylinder surfaces.

Streaky structures are clearly visible all over the surfaces including the narrow part of the duct, where the intensity of streaks is, however, significantly lower than that in the wide part.

\subsection{Secondary motion}

Anisotropy of Reynolds stresses in the circumferential direction causes the appearance of a secondary motion in the cross-sectional plane of the duct. The streamlines of the secondary motion are shown in figure 14(a). The secondary motion exhibits a pair of main well-pronounced counter-rotating vortices on each side of the plane of symmetry. These vortices transfer the high-momentum fluid from the wide to the narrow parts of the duct along the midline of the gap and return the slow-momentum fluid along the duct walls. The vortex attached to the inner cylinder is rather more intense compared to its partner attached to the outer cylinder. The maximum velocity in the secondary motion is 0.0135 and is attained in the vicinity of the inner wall. Contour map of secondary-motion intensity $\sqrt{U_{\xi}^{2}+U_{\eta}^{2}}$ is shown in figure $14(b)$. 

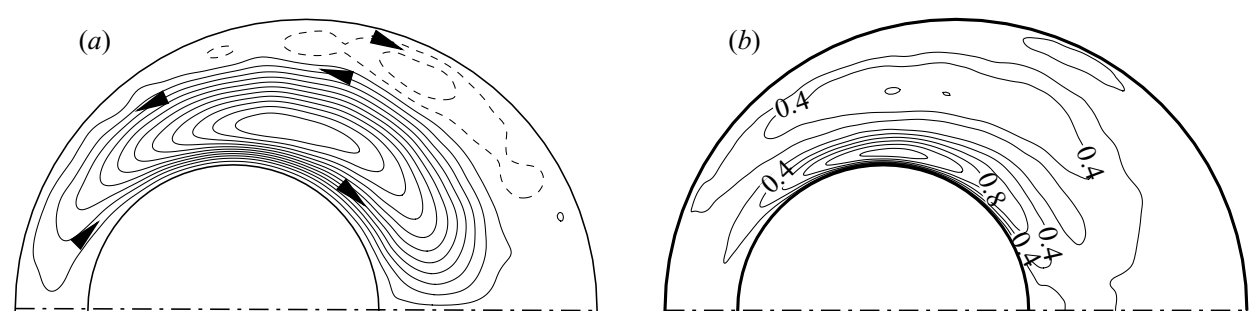

FiguRE 14. (a) Streamlines of secondary motion and $(b)$ its intensity $\sqrt{U_{\xi}^{2}+U_{\eta}^{2}} \times 100$. Solid and dashed streamlines correspond to a counter-clockwise and a clockwise rotation, respectively.

Similar secondary-motion pattern with two circulation cells on each half of the duct with the larger vortex located close to the inner wall was detected experimentally by Nouri et al. (1993). They reported the secondary-motion value of $2.5 \%$ of the bulk velocity in the duct with an eccentricity of 1.0 which was $35 \%$ greater than that with an eccentricity of 0.5 . However, the direction of fluid rotation in the secondary-motion cells measured by Nouri et al. (1993) suggests transport of fluid from wider to narrow regions along the duct walls which is opposite to ours. It should be noted, that direction of secondary motion measured by Nouri et al. (1993) is atypical to turbulent flows in non-circular ducts. The existing experimental and DNS data for ducts with rectangular (Gavrilakis 1992; Nikitin 1997) and elliptic (Nikitin \& Yakhot 2005; Voronova \& Nikitin 2007) cross-section point to the opposite direction of secondary motion with fluid transport from the wider part to the narrow along the middle of the gap and return transport along the walls.

In experiments of Kacker (1973) only one circulation cell was detected on each half of the eccentric duct cross-section. It is attached to the inner cylinder and is similar to our largest cell of the two. It should be noted that the experiments of Kacker (1973) were conducted in the duct with diameter ratio $\gamma=0.18$ which is very different from ours $\gamma=0.5$. It is also possible that the smaller circulation cell was not detected in Kacker (1973) because of its small intensity.

The secondary-motion pattern shown in figure 14(a) was obtained by the averaging of the flow in time, along the streamwise direction and about the symmetry plane $y=0$. As it was noted in the context of Reynolds stresses, the time-averaging interval $T_{a v}=2817$ is not fully adequate for the characteristics reflecting azimuthal inhomogeneity of the flow. It is unlikely, however, that the smaller circulation cell in the secondary motion appeared as a result of the averaging procedure or inadequacy of the time-averaging interval. Figure 15 presents secondary-motion patterns obtained with no averaging over the plane $y=0$ and with two very different time-averaging intervals. One can see from the figure that in spite of some difference in fine details, the main pattern with two circulation cells in each half of the duct cross-section is represented well in both cases.

\subsection{On the forces acting on the duct walls}

The presence of the secondary motion implies the existence of a non-zero pressure force

$$
F_{P, x}=-\int_{0}^{2 \pi} P(\boldsymbol{n}, \boldsymbol{i}) \lambda \mathrm{d} \eta
$$



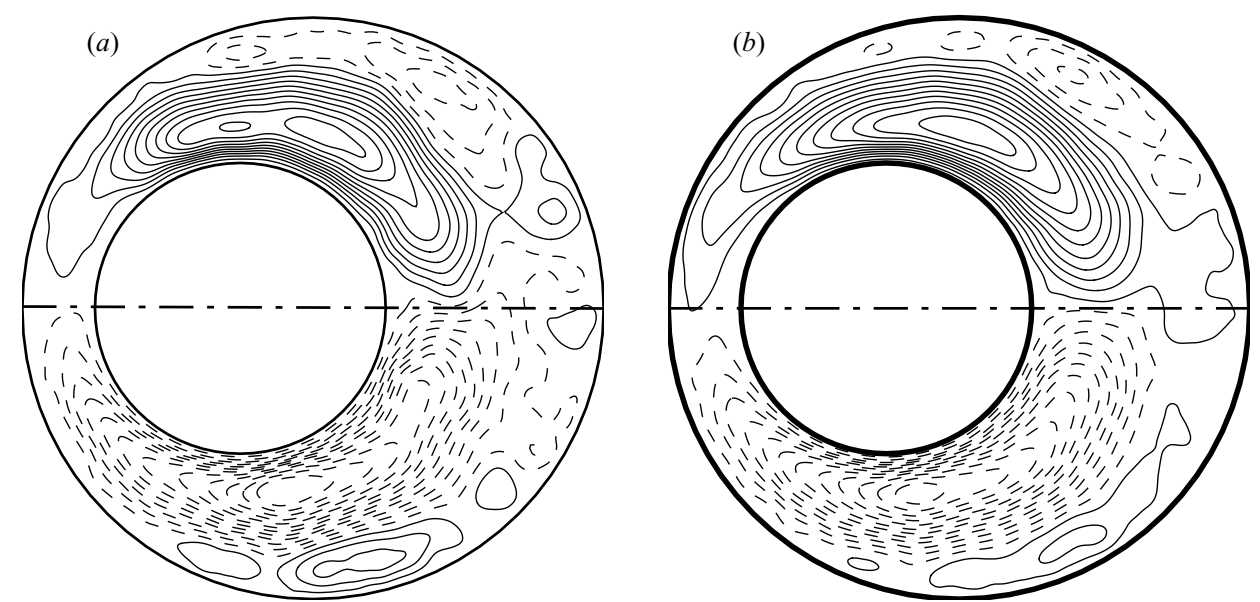

FIGURE 15. Streamlines of secondary motion with no averaging about symmetry plane $y=0$. Time-averaging intervals: (a) $T_{a v}=1045 ;(b) T_{a v}=2817$.
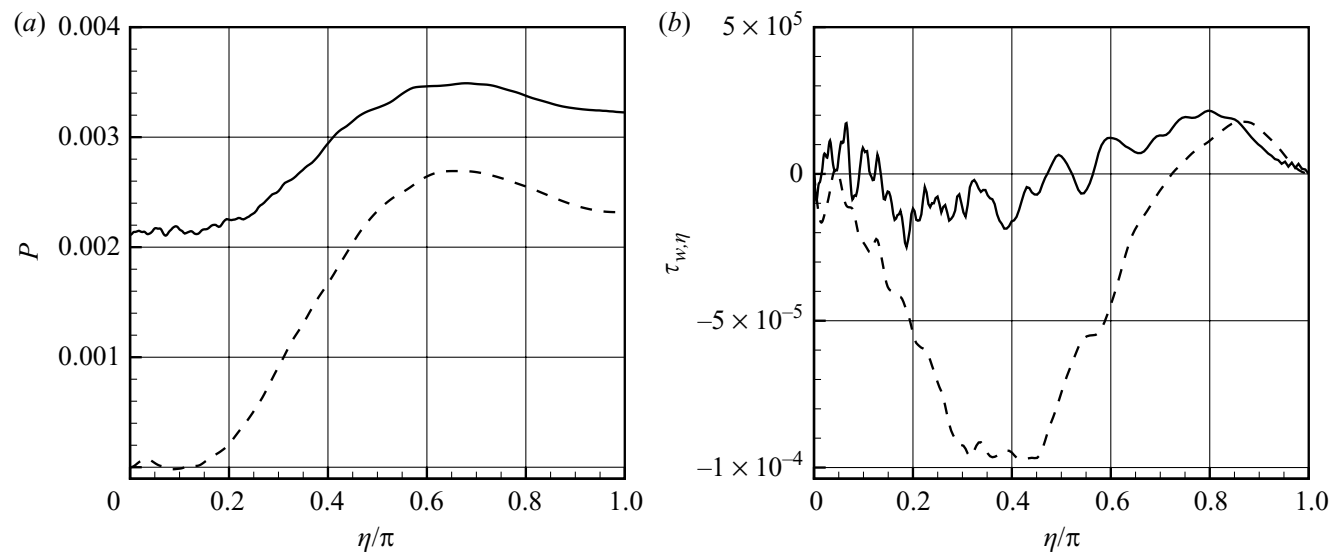

Figure 16. (a) Pressure distribution on the cylinders wall; $(b)$ circumferential component of the wall shear stress. Dashed lines: inner cylinder; solid lines: outer cylinder.

acting on each of the cylinders. From the $\eta$-component of the momentum equation it follows that on the cylinder surfaces

$$
\frac{\partial P}{\partial \eta}=\rho \nu \lambda^{-1} \frac{\partial^{2} U_{\eta}}{\partial \xi^{2}} .
$$

Thus, the inhomogeneity of the pressure distribution around the duct walls is caused by the presence of the secondary motion. The main secondary-motion pattern shown in the figure 14 suggests that $\partial^{2} U_{\eta} / \partial \xi^{2}$ is positive on a large part of both cylinders, which means that the wall pressures on both cylinders in the narrow part of the duct is greater than those in the wide part. Integration of (3.5) supports this conjecture. Pressure distribution on the cylinders wall is shown in figure 16(a). A free constant of the integration is chosen so that $P=0$ on the inner cylinder at $\eta=0$. It is readily seen from figure 16(a) that the pressure force acts on the cylinders in the opposite directions. 
Apart from the pressure force there is a friction force caused by the circumferential component of the wall shear stress $\tau_{w, \eta}=\rho v \partial U_{\eta} / \partial n$ on the cylinders surfaces. In the last expression $n$ stands for the normal vector pointing from the rigid wall into the fluid. It is readily seen from the figure $14(a)$ that $\tau_{w, \eta}$ is negative on the inner wall, i.e. the friction force acts on the inner cylinder from narrow gap towards the wide gap. Concerning the outer cylinder, there are regions of both negative and positive shear stress. The distribution of $\tau_{w, \eta}$ along the inner and outer walls is shown in figure $16(b)$. By comparing with the axial component of the wall shear stress $\tau_{w, z}$ shown in the figure 4 one can see that the maximum of the circumferential component is about $2 \%$ of the axial component and is attained on the inner cylinder. The integral friction force due to $\tau_{w, \eta}$ acting on each cylinder (per unit length) is directed along the $x$-axis ( $y$-component of the force is zero because of the symmetry over the plane $y=0)$ and is expressed by the integral

$$
F_{\tau, x}=\int_{0}^{2 \pi} \tau_{w, \eta}\left(\boldsymbol{k}_{\eta}, \boldsymbol{i}\right) \lambda \mathrm{d} \eta,
$$

where $\boldsymbol{i}$ and $\boldsymbol{k}_{\eta}$ are the unit vectors in the $x$ and $\eta$ directions, respectively. $F_{\tau, x}$ is estimated as $2 \times 10^{-4}$ for the inner cylinder and $5 \times 10^{-5}$ for the outer one. For comparison, the friction force $F_{\tau, z}$ acting in the streamwise direction due to the axial component of the wall shear stress is equal to $2.7 \times 10^{-2}$ for the inner and $5.1 \times 10^{-2}$ for the outer cylinder.

In aggregate, the forces $F_{x}=F_{\tau, x}+F_{P, x}$ acting on the inner and the outer cylinder are about $\pm 4.5 \times 10^{-3}$. The forces act on the cylinders in the opposite directions along the $x$-axis tending to eliminate the eccentricity of the duct.

Probably, the estimation of friction force acting on the outer cylinder is not accurate, because the time-averaging interval used in the paper is not fully adequate for settling the secondary flow in the vicinity of the outer cylinder. Most importantly, however, this value is in any case much less than the pressure forces (by 2 orders of magnitude) and thus it cannot change the overall conclusion about the direction of forces acting on the cylinders.

\section{Thermal field quantities}

\subsection{Mean temperature}

Mean temperature distribution over the duct's cross-section is shown in figure 17 $(a)$ in the form of contour map and in figure 17(b) in the form of radial profiles. Mean flow isotherms are close to circular coordinate lines $\xi=$ constant, as these are in the laminar flow, where temperature is presented by the expression $T(\xi, \eta)=\left(\xi-\xi_{1}\right) /\left(\xi_{2}-\xi_{1}\right)$. However, dependence of the mean temperature on the $\xi$ variable in turbulent regime is strongly nonlinear, as it is seen in the figure $17(b)$.

The average temperature in the flow is $T_{b}=0.65\left(T_{b}=0.63\right.$ in the laminar flow). Defining Nusselt number as $N u=D_{h} H / \kappa, H=Q / S\left|T_{w}-T_{b}\right|$, where $H$ is the heat transfer coefficient, $D_{h}=2 \delta$ is the hydraulic diameter, $Q$ is the heat flux, $T_{w}$ is the wall temperature and $S$ is the surface area of respective wall, one obtains $N u_{1}=24.2$ and $N u_{2}=22.6$ for the inner and outer walls, respectively. Corresponding values in the laminar flow are 5.35 and 4.47. The local Nusselt numbers $N u_{\text {local }}(\eta)$ on the inner and outer duct walls are defined by using the local heat fluxes and local duct clearance $h(\eta)$ as a length scale. $N u_{\text {local }}(\eta)$ distributions are shown in figure 18(a). On the both walls the local Nusselt numbers decrease monotonically with $\eta$ from the 
(a)
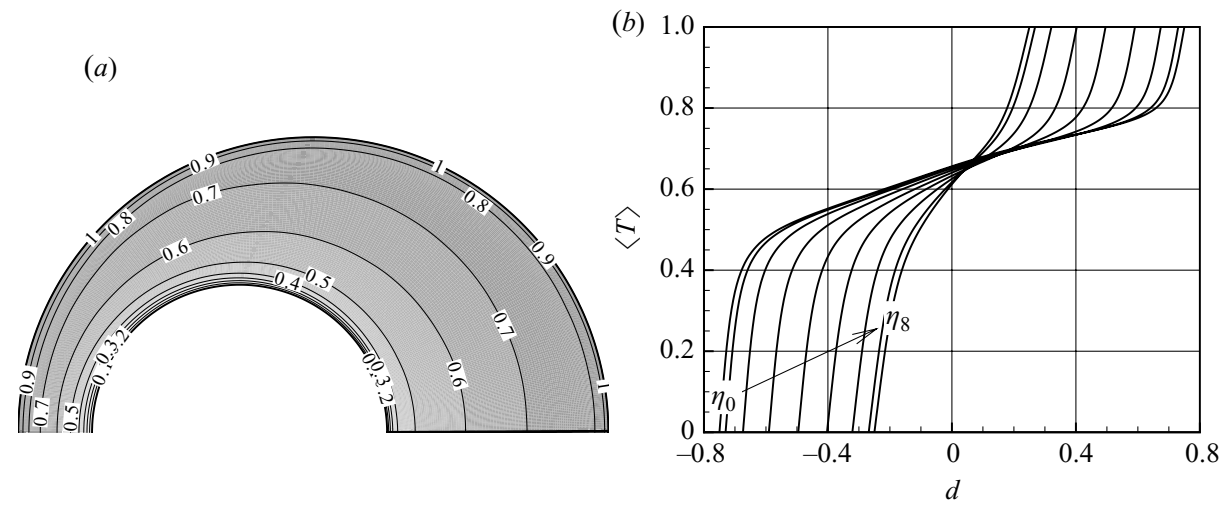

FIGURE 17. (a) Mean temperature contours; $(b)$ mean temperature profiles.
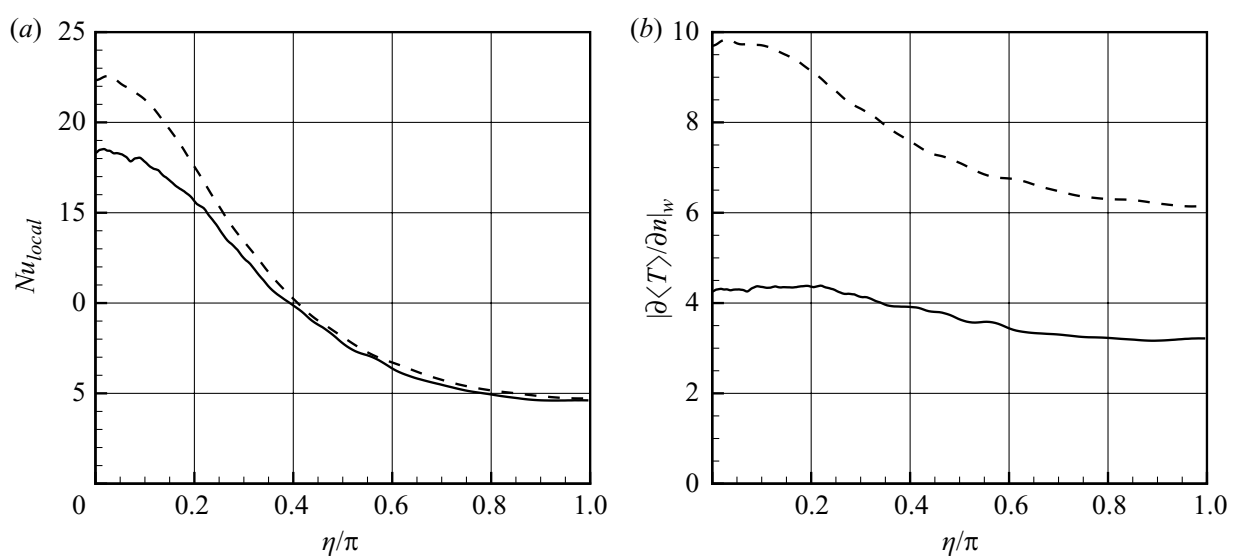

FIGURE 18. (a) Local Nusselt number; $(b)$ wall temperature gradient. Dashed line: inner cylinder; solid line: outer cylinder.

values close to the corresponding global Nusselt numbers down to the values in the laminar flow. Thus, the flow in the narrowest gap may be considered as laminar in terms of the local Nusselt number. However, as it will be shown below, this region cannot be considered as laminar in terms of the intensity of temperature fluctuations.

Figure $18(b)$ presents circumferential variation of the temperature gradient on the cylinder walls. Because of the imposed streamwise periodicity of the temperature field and isothermal boundary conditions on the cylinder walls, the mean heat fluxes are the same on both walls. Since the surface area of the outer boundary is twice as that of the inner one, on average the local heat flux and wall temperature gradient on the inner wall are twice as large as they are on the outer wall. As it is seen in the figure $18(b)$ this ratio holds approximately at each $\eta$. Mean temperature gradient in the narrow gap is on average three times larger than it is in the wide gap. Nevertheless, the wall gradient is maximal in the wide gap on the both cylinders. The small value of average gradient in the wide gap is due to the temperature plateau between the wall regions (see figure $17 b$ ).

\subsection{Temperature fluctuations}

Radial profiles of temperature variance $\left\langle T^{\prime} T^{\prime}\right\rangle$ and of velocity-temperature correlations $\left\langle T^{\prime} u_{i}^{\prime}\right\rangle$ are shown in figure 19. For each $\eta$, the temperature variance 

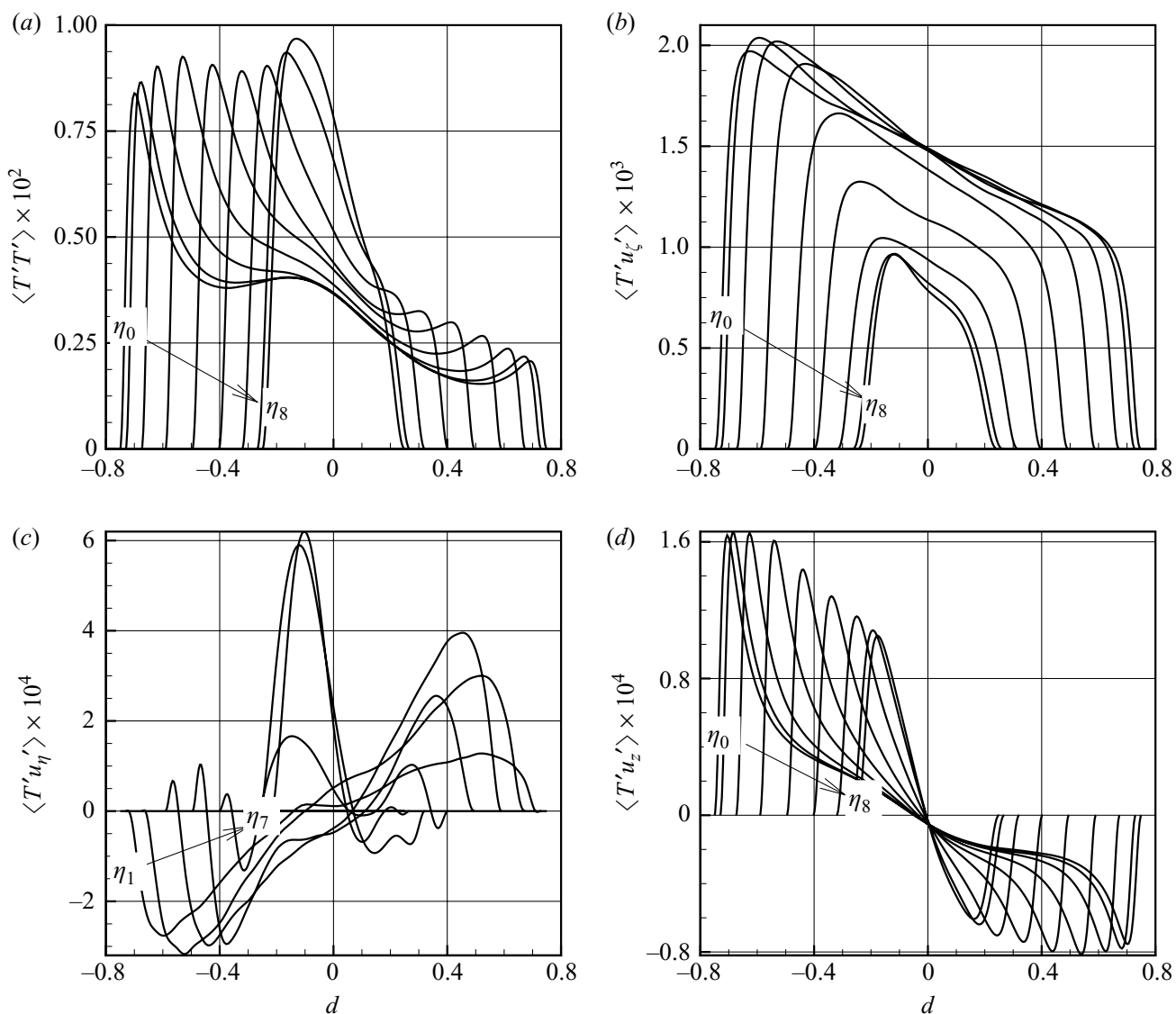

FIGURE 19. Profiles of temperature variance and velocity-temperature correlations.

(and the intensity of temperature fluctuations $T_{r m s}$, which is $\left\langle T^{\prime} T^{\prime}\right\rangle^{0.5}$ ) near the inner cylinder wall is significantly higher than that near the outer wall. In the wide-gap region, temperature fluctuations are about two times more intense near the inner wall than those near the outer one. This suggests that the main mechanism of temperature fluctuations in the flow is the action of radial velocity fluctuations across the mean temperature gradient which is twice as steep in the inner wall region as in the outer wall one. The main production term in the equation for the temperature variance is $-\left\langle T^{\prime} u_{\xi}^{\prime}\right\rangle G_{T}$, where $G_{T}=\lambda^{-1} \partial\langle T\rangle / \partial \xi$ is the mean temperature gradient in the wallnormal direction. In the wide gap the correlation $-\left\langle T^{\prime} u_{\xi}^{\prime}\right\rangle$ in the inner-wall region is two times higher than that in the outer-wall region (see figure 19b). This is in agreement with the amplitude ratio of the temperature fluctuations (figure 19a) and $u_{\xi}$-velocity fluctuations (figure $10 a$ ) in the inner- and outer-wall regions.

Another fact worth to be mentioned is that the intensity of temperature fluctuations is anomalously high in the narrow gap compared with that in the wide gap, taking in account relatively low-production term $-\left\langle T^{\prime} u_{\xi}^{\prime}\right\rangle G_{T}$ in the narrow-gap region. It may be speculated that the reason for this disproportion consists in the following. In the wide-gap region the temperature fluctuations being produced near the wall are then transferred away from the wall where the mean-temperature gradient is not so steep and conditions for the production of temperature fluctuations are not so favourable. Whereas in the narrow gap, the temperature fluctuations being produced 
on the background of steep gradient are accumulated in the same place with less transfer to other flow regions.

The shape of $\left\langle T^{\prime} u_{z}^{\prime}\right\rangle$-correlation profiles (figure 19d) confirms the suggestion that in the background of the temperature- and $u_{z}$-fluctuations lies in the same mechanism, with heat and momentum transfer due to wall-normal velocity fluctuations acting across the temperature and mean velocity gradients. The maxima of $\left|\left\langle T^{\prime} u_{z}^{\prime}\right\rangle\right|$ near each wall are proportional to $T_{r m s} u_{z, r m s}$. Correlation coefficient $\rho\left(T, u_{z}\right)=$ $\left\langle T^{\prime} u_{z}^{\prime}\right\rangle /\left(T_{r m s} u_{z, r m s}\right)$ is as large as \pm 0.95 near the inner and outer walls, respectively.

\section{Summary and discussion}

A direct numerical simulation of a turbulent flow in an eccentric annular duct with diameter ratio $\gamma=0.5$ and eccentricity $e=0.5$ was carried out with $128 \times 512 \times 256$ mesh points at a Reynolds number of 8000 , based on the bulk velocity and hydraulic diameter. A second-order finite-difference conservative scheme is used for the spatial discretization in conjunction with a third-order time advancement scheme.

Simulation results are in overall qualitative and quantitative agreement with the existing experimental data. The value of friction factor obtained in simulation coincides with the friction factor for a circular pipe as predicted by the Blasius formula. This is in agreement with the Nouri et al. (1993) prediction for the eccentric duct. Mean velocity profiles agree very well with measurements of Nouri et al. (1993) both in the wide and narrow gaps. When mean velocity and distance to the wall are normalized by the wall variables defined by the local wall shear stress, the logarithmic behaviour in the mean velocity profiles is visible only in the wide-gap region, whereas in the narrow-gap region mean velocity profiles lie significantly higher. In the widegap region, where the logarithmic layer can be detected in the mean velocity profiles, the inner profiles lie substantially lower than the outer ones. These observations agree with experimental findings of Jonsson \& Sparrow (1966) and Nouri et al. (1993).

Anisotropy of Reynolds stresses in the circumferential direction causes the appearance of a secondary motion in the cross-sectional plane of the duct. The secondary motion obtained in the present simulation exhibits a pair of main wellpronounced counter-rotating vortices on each side of the plane of symmetry. The maximum velocity in the secondary motion is 0.0135 and is attained in the vicinity of the inner wall. The secondary-motion pattern of the present study is similar to that of Nouri et al. (1993). However, the direction of fluid rotation reported in Nouri et al. (1993) is opposite to present results. We cannot give any rational explanation of this discrepancy apart of noting that direction of secondary motion reported in Nouri et al. (1993) disagrees with general form of known turbulent secondary motions in non-circular ducts. Secondary motion reported by Kacker (1973) exhibits only one circulation cell in each half of the eccentric duct cross-section, which resembles the largest cell of our simulation. In our opinion this disagreement can be explained by the difference in the flow geometry or inability of experimental detection of the extremely low-amplitude motion in the smaller circulation cell.

Even in the narrowest part of the annulus the calculated flow has many features of a turbulent flow: the mean profile is more filled than the laminar parabolic profile, the distributions of the Reynolds stresses are mostly of turbulent character, there are streaks, sweeps dominate ejections near the wall and vice versa away from the wall. However, in the narrowest part of the annulus the local Reynolds number based on the wall shear stress is below the value for which the flow can be turbulent in a plane channel. Hence, in this part of the annulus turbulence can be expected to 
be not self-sustaining. One possible explanation of the physical mechanism by which turbulence is sustained in the narrowest part is suggested by figure $10(b)$. As can be seen from this figure, the amplitude of the circumferential velocity fluctuations is anomalously large in the narrow part of the channel. When a volume of fluid is in the narrowest area, turbulence recedes. However, before it decays completely, a large circumferential fluctuation moves this volume to the neighbouring wider part of the annulus, where turbulence recovers since it can sustain itself there. While other physical mechanisms of sustaining turbulence in the narrow part of the annulus can probably be suggested, one can conclude that even though the flow considered has no laminar regions, it will present a considerable challenge for semi-empirical turbulence modelling.

The present study revealed a large number of flow details which have not been documented so far. In particular, the components of the Reynolds stress tensor, temperature-velocity correlations and some others were obtained for the first time for such kind of a flow. The results of the paper will serve as a benchmark test case for turbulence modelling, specifically for models intended to be used for flows with partly turbulent regimes.

This research was supported by EPSRC under grant EP/D050871/1. The work of NN was supported also by the Russian Foundation for Basic Research under grant 08-01-00489-a.

\section{REFERENCES}

Bradshaw, P. 1987 Turbulent secondary flows. Ann. Rev. Fluid Mech. 19, 53-74.

Brodkey, R. S., Wallace, J. M. \& Eckelmann, H. 1974 Some properties of truncated turbulence signals in bounded shear flows. J. Fluid Mech. 63, 209-224.

Deissler, R. G. \& TAYLOR, M. F. 1955 Analysis of fully developed turbulent heat transfer and flow in an annulus with various eccentricities. NACA TN, No. 3451.

Demuren, A. O. \& RoDI, W. 1984 Calculation of turbulence-driven secondary motion in non-circular ducts. J. Fluid Mech. 140, 189-222.

Dodge, N. A. 1963 Friction losses in annular flow. Paper No. 63-WA-11, The American Society of Mechanical Engineers.

Eggels, J. G. M., Unger, F., Weiss, M. H., Westerweel, J., Adrian, R. J., Friedrich, R. \& Nieuwstadt, F. T. M. 1994 Fully developed turbulent pipe flow: a comparison between direct numerical simulation and experiment. J. Fluid Mech. 268, 175-209.

GaVRILAKIS, S. 1992 Numerical simulation of low-Reynolds-number turbulent flow through a straight square duct. J. Fluid Mech. 244, 101-129.

GILBERT, N. \& KLEISER, L. 1991 Turbulence model testing with the aid of direct numerical simulation results. In Proceedings of 8th Symposium on Turbulent Shear Flows, Munich, September 9-11, 1991, Paper 26-1.

Huser, A. \& Biringen, S. 1993 Direct numerical simulation of turbulent flow in a square duct. J. Fluid Mech. 257, 65-95.

Jonsson, V. K. \& Sparrow, E. M. 1966 Experiments on turbulent-flow phenomena in eccentric annular ducts. J. Fluid Mech. 25, 65-86.

KACKER, S. C. 1973 Some aspects of fully developed turbulent flow in non-circular ducts. J. Fluid Mech. 57, 583-602.

Kim, J., Moin, P. \& Moser, R. 1987 Turbulence statistics in fully developed channel flow at low Reynolds number. J. Fluid Mech. 177, 133-166.

Moin, P. \& MAHESH, K. 1998 Direct numerical simulation: a tool in turbulence research. Ann. Rev. Fluid Mech. 30, 539-578.

Nikitin, N. 1994 Direct numerical modelling of three-dimensional turbulent flows in pipes of circular cross section. Fluid Dyn. 29, 749-757.

Nikitin, N. 1996 Statistical characteristics of wall turbulence. Fluid Dyn. 31, 361-370. 
Nikitin, N. 1997 Numerical simulation of turbulent flows in a pipe of square cross-section. Phys.Dok. 42, 158-162.

Nikitin, N. $2006 a$ Direct simulation of turbulent flow in eccentric pipes. Comp. Maths Math. Phys. 46, 489-504.

Nikitin, N. $2006 b$ Finite-difference method for incompressible Navier-Stokes equations in arbitrary orthogonal curvilinear coordinates J. Comput. Phys. 217, 759-781.

Nikitin, N. \& Yакнот, A. 2005 Direct numerical simulation of turbulent flow in elliptical ducts. J. Fluid Mech. 532, 141-164.

Ninokata, H., Okumura, T., Merzari, E. \& Kano, T. 2006 Direct numerical simulation of turbulent flows in an eccentric annulus channel. Annual Report of the Earth Simulator Center, Earth Simulator Center pp. 293-297.

Nouri, J. M., Umur, H. \& Whitelaw, J. H. 1993 Flow of Newtonian and non-Newtonian fluids in concentric and eccentric annuli. J. Fluid Mech. 253, 617-641.

Ogino, F., Sakano, T. \& Mizushina, T. 1987 Momentum and heat transfers from fully developed turbulent flow in an eccentric annulus to inner and outer tube walls. Heat Mass Transfer 21, 87-93.

SpalaRT, P. R. 1988 Direct simulation of a turbulent boundary layer up to $R e_{\theta}=1410$. J. Fluid Mech. 187, 61-98.

SwarzTrauber, P. N. 1974 A direct method for the discrete solution of separable elliptic equations. SIAM J. Numer. Anal. 11, 1136-1150.

Tsukahara, T., Seki, Y., Kawamura, H. \& Tochio, D. 2005 DNS of turbulent channel flow at very low Reynolds numbers. In Proceedings of the Fourth International Symposium on Turbulence and Shear Flow Phenomena, Williamsburg, VA, June 27-29, Taylor \& Frances LTD, pp. 935-940.

Usui, H. \& Tsuruta, K. 1980 Analysis of fully developed turbulent flow in an eccentric annulus. J. Chem. Engng Jpn. 3, 445-450.

Voronova, T. \& Nikitin, N. 2007 Results of direct numerical simulation of turbulent flow in a pipe of elliptical cross-section. Fluid Dyn. 42, 201-211.

Willmarth, W. W. \& Lu, S. S. 1972 Structure of the Reynolds stress near the wall. J. Fluid Mech. 55, 65-92.

Wilson, J. T. 1978 Fully developed laminar incompressible flow in an eccentric annulus. AIChE 24 (4), 733-735.

WolfFe, R. A. 1962 Axial turbulent flow in a circular pipe containing a fixed eccentric core. M.S. Thesis, Lehigh University, Bethlehem, PA. 